\title{
Distribuição geográfica de Psychosaura agmosticha (Rodrigues, 2000) (Squamata, Mabuyidae)
}

\author{
Arnaldo José Correia Magalhães Júnior ${ }^{1 *}$ \\ Luiz Cezar Machado Pereira ${ }^{2,3}$ \\ Patrícia Avello Nicola ${ }^{2,3}$ \\ Leonardo Barros Ribeiro ${ }^{2,3}$ \\ Severino Mendes de Azevedo Júnior ${ }^{3}$ \\ ${ }^{1}$ Universidade Federal do Vale do São Francisco, Colegiado Acadêmico de Ciências da Natureza \\ Rua João Ferreira dos Santos, s/n, CEP 64.770-000, São Raimundo Nonato, PI - Brasil \\ ${ }^{2}$ Universidade Federal do Vale do São Francisco, Colegiado Acadêmico de Ciências Biológicas \\ Petrolina, PE - Brasil \\ ${ }^{3}$ Centro de Conservação e Manejo de Fauna (CEMAFAUNA - Caatinga), Petrolina - PE, Brasil \\ ${ }^{4}$ Universidade Federal Rural de Pernambuco, Departamento de Biologia, Recife - PE, Brasil \\ *Autor para correspondência \\ arnaldo.magalhaes@univasf.edu.br
}

Submetido em 12/04/2013

Aceito para publicação em 06/03/2014

\section{Resumo}

Durante as atividades de resgate de fauna do Projeto de Integração do Rio São Francisco com as bacias do nordeste setentrional foram capturados 39 exemplares de Psychosaura agmosticha (Rodrigues, 2000) em localidades nos estados do Ceará, Paraíba e Pernambuco, região Nordeste do Brasil. Relacionamos estes registros aos encontrados na literatura e constatamos a espécie em quatro formações fitofisionômicas distintas: Dunas arenosas do rio São Francisco, caatinga arbustiva, caatinga arbórea e formações de florestas residuais e ecotonais. A ampliação da distribuição geográfica e os novos registros de ocorrência de P. agmosticha, bem como sua ocorrência em diferentes formações fitofisionômicas da Caatinga e em áreas de florestas residuais e ecótonos, demonstram que, apesar de sua distribuição geográfica ser mais ampla do que se conhecia, seu padrão de distribuição relictual ainda se mantém, em virtude da ocorrência de $P$. agmosticha estar relacionada à ocorrência de bromélias. Contudo, o endemismo da espécie para o bioma Caatinga parece não ser absoluto.

Palavras-chave: Caatinga; Lagartos; Mabuya; Nordeste do Brasil

\section{Abstract}

Geographic distribution of Psychosaura agmosticha (Rodrigues, 2000) (Squamata, Mabuyidae). During fauna rescue activities of the integration project of the São Francisco River and other basins of the northern part of Northeast Brazil, 39 individuals of Psychosaura agmosticha (Rodrigues, 2000) were collected in the states of Ceará, Paraíba and Pernambuco, located in Northeast Brazil. We compared our recordings with those found in the literature and observed that P. agmosticha can occur in four different phytophysiognomies: sandy dunes of the San Francisco River, shrubby caatinga, arboreal caatinga, and forest remnants and ecotone 
formations. In spite of the expansion of the known geographic distribution and new records of P. agmosticha, the species retains its character of relictual distribution, due to its occurrence being related to the presence of bromeliads. Furthermore, we suggest that this species is not totally endemic to the caatinga biome.

Key words: Caatinga; Lizards; Mabuya; Northeast Brazil

\section{Introdução}

No Brasil, as 14 espécies conhecidas para a família Mabuyidae estavam agrupadas em dois gêneros, Mabuya Fitzinger, 1826 e Trachylepis Fitzinger, 1843 (BÉRNILS; COSTA, 2012). Recentemente este grupo sofreu ampla revisão taxonômica, na qual 13 espécies ocorrentes no Brasil, outrora agrupadas no gênero Mabuya, foram rearranjadas em nove gêneros: Aspronema, Brasiliscincus, Copeoglossum, Exila, Manciola, Notomabuya, Panopa, Psychosaura e Varzea (HEDGES; CONN, 2012). As espécies deste grupo habitam áreas com os mais variados tipos de clima e vegetação, como as florestas Atlântica e Amazônica, os cerrados e as caatingas (FREITAS; SILVA, 2006). Na região Nordeste do Brasil existem seis espécies deste grupo, quatro delas ocorrendo nos domínios das Caatingas (RODRIGUES, 2000). Destas, Brasiliscincus heathi (Schmidt \& Inger, 1951) é considerada de ampla ocorrência e Psychosaura macrorhyncha (Hoge, 1947), Copeoglossum arajara (Rebouças-Spieker, 1981) e Psychosaura agmosticha (Rodrigues, 2000) são consideradas de ocorrência restrita ou relictual, sendo esta última endêmica do bioma Caatinga (RODRIGUES, 2000).

Psychosaura agmosticha pode ser diagnosticado pela presença de um par de escamas frontoparietais, quatro supraoculares, corpo cilíndrico e alongado, focinho alongado e presença de duas linhas negras laterais que se estendem até aproximadamente metade do corpo, hábitos diurnos e é encontrada, sobretudo nas Caatingas com predominância de bromélias (RODRIGUES, 2000).

Esta comunicação tem por objetivo contribuir acerca do conhecimento da distribuição geográfica de $P$. agmosticha na região Nordeste do Brasil, indicando as formações de ocorrência da espécie e discutindo alguns aspectos quanto ao seu padrão de distribuição.

\section{Material e Métodos}

O presente estudo foi conduzido com base na análise de indivíduos capturados ou coletados durante dois momentos distintos: a) atividades de resgate de fauna e visitas técnicas do Projeto de Integração do Rio São Francisco (PISF) com as bacias do nordeste setentrional e b) levantamento de fauna do Ramal do Agreste, ambos no período de janeiro de 2007 a janeiro 2009, de acordo com as licenças emitidas pelo IBAMA, no 042/2007 e 125.r/2010 (Processo no 02001.003112/2007-12).

A coleta dos exemplares foi realizada entre janeiro de 2007 e janeiro de 2009, com emprego dos métodos detalhados a seguir:

A) Armadilhas de interceptação e queda com cercasguia: Armadilhas contendo recipientes enterrados no solo (baldes plásticos) e interligados por cercas-guia (CENCHIN; MARTINS, 2000). Foram instaladas quatro armadilhas equidistantes $30 \mathrm{~m}$ nas áreas de influência direta do Projeto de transposição do rio São Francisco com as Bacias hidrográficas do nordeste setentrional, nos estados do Ceará, Pernambuco e Paraíba. Cada armadilha era composta por quatro baldes de $25 \mathrm{~L}$, dispostos em linha e interligados por cercas guias de lona com cinco metros de comprimento e um metro de altura.

B) Busca ativa: Foram inventariados transectos aleatórios nas áreas estudadas. As visitas foram realizadas diariamente entre as $09 \mathrm{~h} 00 \mathrm{~min}$ e 16h00min, onde foram vistoriados, troncos em decomposição, cavidades de troncos, rochas, a vegetação, e outros locais que podem servir de abrigo para esses animais.

Os espécimes coletados foram depositados na Coleção Herpetológica do Museu de Fauna da Caatinga (MFCH), do Centro de Conservação e Manejo da Fauna da Caatinga (CEMAFAUNA) da Universidade Federal 
do Vale do São Francisco e na Coleção Herpetológica da Universidade Federal de Pernambuco - UFPE. Para cada indivíduo capturado foram obtidas informações referentes ao local, data de coleta, morfometria, posicionamento geográfico e características da vegetação da área amostrada.

\section{Resultados e Discussão}

Ao todo, 39 indivíduos de Psychosaura agmosticha foram capturados. Destes, foi possível obter informações morfométricas de 25 (Tabela 1).

Psychosaura agmosticha foi registrada em nove municípios de três estados (Tabela 2), sendo os registros de Mauriti, Milagres e Brejo Santo, os primeiros para o estado do Ceará, ampliando sua distribuição geográfica (Figura 1).

Além dos novos registros de ocorrência e a ampliação da distribuição geográfica de $P$. agmosticha, a análise dos tipos de vegetação das localidades em que a espécie foi registrada indicou a presença de $P$. agmosticha em quatro formações distintas do bioma Caatinga: Dunas do Rio São Francisco, caatinga arbórea, caatinga arbustiva e formações de ecótonos e florestas residuais semelhantes a brejos de altitude. Estes registros, ampliam os tipos de formações de ocorrência da espécie, até então registradas para apenas duas formações fitofisionômicas da Caatinga: Caatinga arbóreo-arbustiva e arbórea (RODRIGUES, 2000; ARZABE et al., 2005) e recentemente em restingas de Floresta Atlântica na Bahia (DIAS; ROCHA, 2013).

Verificamos ainda que a ocorrência de P. agmosticha esteve sempre associada à presença de bromélias das espécies Bromelia laciniosa e Encholirium spectabile, ambas distribuídas nos afloramentos rochosos das áreas de Caatinga estudadas, corroborando Rodrigues (2000). Dias e Rocha (2013) também registraram a espécie associada a ocorrências de bromélias de duas espécies

TABELA 1: Morfometria dos indivíduos (não autotomizados) de Psychosaura agmosticha capturados durante as atividades de resgate de fauna do Projeto de Integração do Rio São Francisco no período de janeiro de 2007 a janeiro de 2009.

\begin{tabular}{|c|c|c|c|c|c|}
\hline Voucher & $\mathrm{CA}(\mathrm{mm})$ & CRC (mm) & CT (mm) & Peso (g) & Localidade \\
\hline MFCH 691 & 8,93 & 66,05 & 141,86 & 8,9 & Floresta - PE \\
\hline MFCH 692 & 9,46 & 72,02 & 163,00 & 7,2 & Floresta - PE \\
\hline MFCH 694 & 8,56 & 64,60 & 135,71 & 6,2 & Floresta - PE \\
\hline MFCH 695 & 7,06 & 55,24 & 99,35 & 5,9 & Floresta - PE \\
\hline MFCH 697 & 8,95 & 63,10 & 123,06 & 5,0 & Floresta - PE \\
\hline MFCH 698 & 9,16 & 62,60 & 158,58 & 4,3 & Floresta - PE \\
\hline MFCH 700 & 6,87 & 54,57 & 91,51 & 4,6 & Floresta - PE \\
\hline MFCH 704 & 4,49 & 29,65 & 71,91 & 4,5 & Custódia - PE \\
\hline MFCH 705 & 9,15 & 67,58 & 147,70 & 5,8 & Floresta - PE \\
\hline MFCH 706 & 9,11 & 63,65 & 142,07 & 4,3 & Custódia - PE \\
\hline MFCH 708 & 8,30 & 51,58 & 124,00 & 6,2 & Sertânia - PE \\
\hline MFCH 709 & 8,04 & 57,72 & 90,67 & 4,4 & Sertânia - PE \\
\hline MFCH 711 & 8,47 & 58,80 & 108,71 & 4,3 & Custódia - PE \\
\hline MFCH 712 & 8,85 & 62,90 & 103,19 & 4,7 & Sertânia - PE \\
\hline MFCH 722 & 9,08 & 65,52 & 145,60 & 5,5 & Petrolândia - PE \\
\hline MFCH 723 & 9,77 & 65,19 & 92,47 & 5,6 & Mauriti-CE \\
\hline UFPE101 & 9,56 & 62,15 & 100,02 & 6,0 & Brejo Santo - CE \\
\hline UFPE102 & 8,20 & 60,12 & 98,50 & 5,5 & Coremas - PB \\
\hline UFPE103 & 8,80 & 63,45 & 139,20 & 4,5 & Arcoverde - PE \\
\hline UFPE104 & 9,82 & 62,05 & 140,20 & 6,6 & Arcoverde - PE \\
\hline MD/DP & $8,53 / 1,23$ & $60,43 / 8,65$ & $120,87 / 26,35$ & $5,5 / 1,18$ & \\
\hline
\end{tabular}

Legenda: CA: comprimento da cabeça; CRC: comprimento rostro-cloacal; CT: comprimento total; MD: Média e DP: Desvio Padrão 
TABELA 2: Localidades, coordenadas geográficas e número de indivíduos de Psychosaura agmosticha capturados por tipo de formação fitofisionômica amostrada durante o estudo.

\begin{tabular}{lccl}
\hline \multicolumn{1}{c}{ Localidades } & Coordenadas & № de indivíduos capturados & \multicolumn{1}{c}{ Tipo de vegetação } \\
\hline PB - Coremas & $6^{\circ} 59^{\prime} \mathrm{S} / 37^{\circ} 56^{\prime} \mathrm{O}$ & 02 & Caatinga Arbórea \\
$\mathrm{PE}$ - Petrolândia & $9^{\circ} 04^{\prime} \mathrm{S} / 38^{\circ} 16^{\prime} \mathrm{O}$ & 04 & Dunas do São Francisco* \\
$\mathrm{PE}$ - Custódia & $8^{\circ} 06^{\prime} \mathrm{S} / 37^{\circ} 38^{\prime} \mathrm{O}$ & 05 & Caatinga Arbórea \\
$\mathrm{PE}$ - Sertânia & $8^{\circ} 04^{\prime} \mathrm{S} / 37^{\circ} 15^{\prime} \mathrm{O}$ & 04 & Caatinga Arbustiva \\
$\mathrm{PE}$ - Floresta & $8^{\circ} 36^{\prime} \mathrm{S} / 38^{\circ} 35^{\prime} \mathrm{O}$ & 10 & Caatinga Arbórea \\
$\mathrm{PE}$ - Arcoverde & $8^{\circ} 25^{\prime} \mathrm{S} / 37^{\circ} 03^{\prime} \mathrm{O}$ & 08 & Floresta resídual* \\
$\mathrm{CE}$ - Mauriti & $7^{\circ} 23^{\prime} \mathrm{S} / 38^{\circ} 46^{\prime} \mathrm{O}$ & 02 & Caatinga Arbórea \\
$\mathrm{CE}$ - Brejo Santo & $7^{\circ} 29^{\prime} \mathrm{S} / 38^{\circ} 59^{\prime} \mathrm{O}$ & 02 & Caatinga Arbórea \\
$\mathrm{CE}$ - Milagres & $7^{\circ} 18^{\prime} \mathrm{S} / 38^{\circ} 56^{\prime} \mathrm{O}$ & 02 & Caatinga Arbórea \\
\hline
\end{tabular}

*Novas formações fitofisionômicas de Caatinga onde $P$. agmosticha foi registrada.

FIGURA 1: Distribuição geográfica de Psychosaura agmosticha: Ceará: 5 - Milagres (nr), 7 - Mauriti (nr) e 8 - Brejo Santo (nr); Rio Grande do Norte: 1 - Santa Maria (JORGE; FREIRE, 2010); Paraíba: 2 - Cacimba de Dentro (ARZABE et al., 2005), 3 Coremas (nr), 6 - Cabaçeiras (RODRIGUES, 2003), 4 - São José dos Cordeiros (FREIRE et al., 2009) e 10 - Sumé (FREIRE et al., 2009); Pernambuco: 11 - Custódia (nr), 12 - Arcoverde (nr), 13 - Sertânia (nr), 14 - Floresta (nr), 15 - Buíque: (MOURA et al., 2011) e 16 - Petrolândia (nr); Alagoas: 17 - Delmiro Gouveia (RODRIGUES, 2000) e 18 - Canindé do São Francisco (RODRIGUES, 2000) e Sergipe: 19 - Itabaiana (CARVALHO et al., 2005) e Bahia: 20 - Jandaíra (DIAS; CARVALHO, 2013).

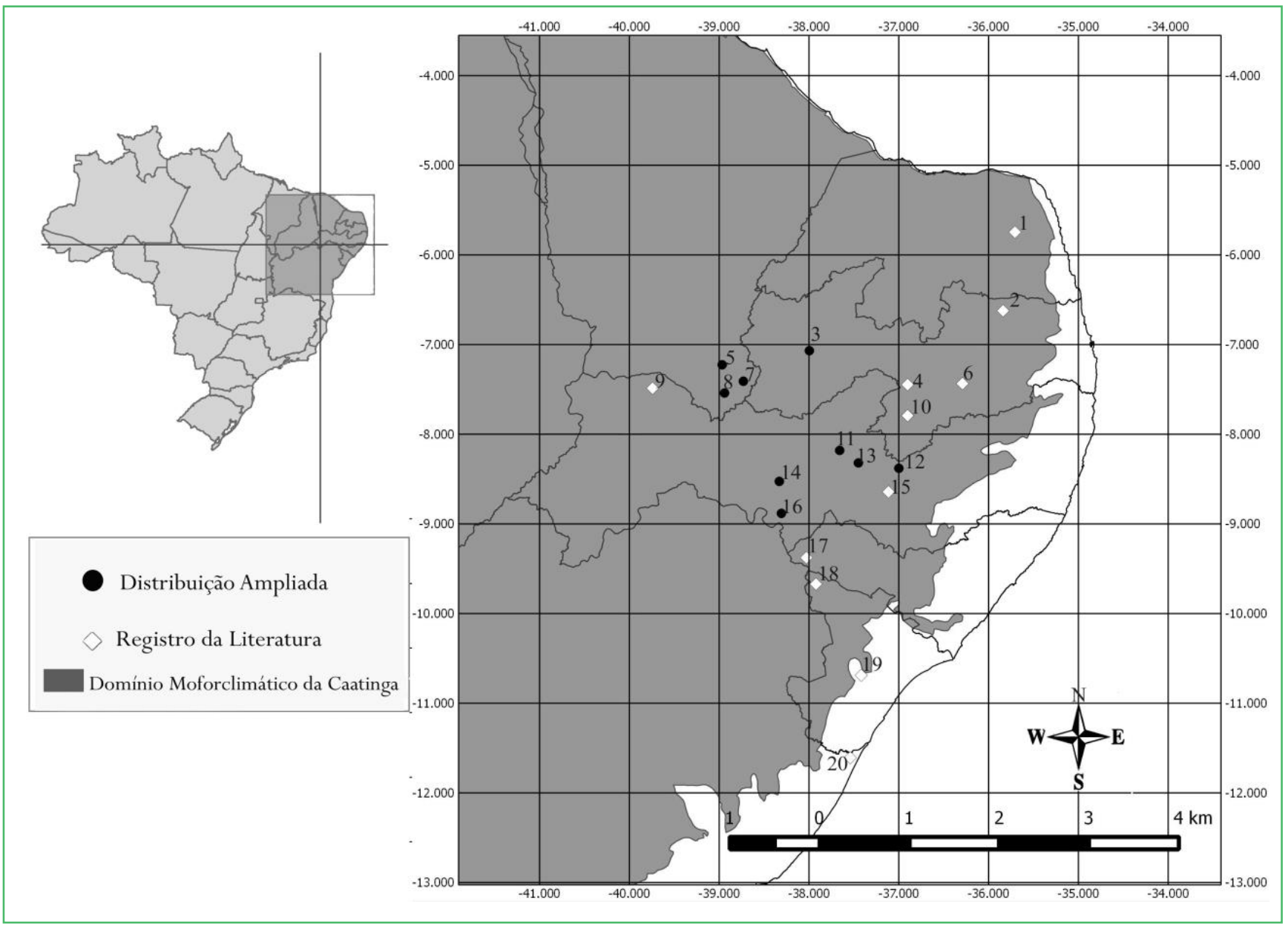


distintas (Aechmera multiflora e Aechmera blanchetiana) nas restingas da Bahia. Este cenário sugere que o padrão de distribuição de $P$. agmosticha é semelhante ao descrito por Vanzolini (1981), para espécies associadas aos refúgios e por Rodrigues (2003) para espécies associadas a formações de dunas arenosas, distinguindo destes apenas face ao fator limitante à ocorrência da espécie ser sua fidelidade ao microhabitat e não uma paisagem. Logo, apesar de ocorrer em muitas localidades da Caatinga e em diferentes fitofisionomias, o padrão de distribuição de $P$. agmosticha configura-se relictual, em face à disponibilidade de seu microhabitat (bromélias).

Destacamos que a ocorrência de $P$. agmosticha em formações de dunas arenosas do rio São Francisco configura uma nova formação registrada para esta espécie. Além das áreas típicas das caatingas, $P$. agmosticha foi encontrada também em formações de florestas residuais em Arcoverde, Pernambuco e áreas de transição com a Floresta Atlântica em Itabaiana, Sergipe (CARVALHO et al., 2005) e em restingas litorâneas da Floresta Atlântica em Jandaíra, Bahia (DIAS; ROCHA, 2013), o que sugere que seu endemismo na Caatinga não é absoluto.

Os novos registros de $P$. agmosticha, incluindo sua ocorrência em diferentes formações fitofisionômicas da Caatinga e em áreas de floresta residual e ecótonos, demonstram que sua distribuição geográfica é mais ampla do que se conhecia. Contudo, o padrão de distribuição relictual, descrito por Rodrigues (2003) para esta espécie, ainda se mantém, ainda que configurado pela relação com seu microhabitat. A despeito disso, o endemismo da espécie para o bioma Caatinga não se sustenta, uma vez que ela foi registrada em áreas de florestas residuais, áreas de transição e recentemente nas restingas litorâneas da Floresta Atlântica (DIAS; ROCHA, 2013).

\section{Agradecimentos}

Ao Ministério da Integração Nacional pela concessão de apoio financeiro para realização da pesquisa; ao Dr. Miguel Trefaut Urbano Rodrigues pelo auxílio na identificação dos exemplares; e ao Dr. Gabriel Skuk Sugliano (in memoriam) pela sua imensurável contribuição à Herpetologia.

\section{Referências}

ARZABE, C.; SKUK, G.; SANTANA, G.; DELFIM, F. R.; LIMA, Y. C. C.; ABRANTES, S. H. F. Herpetofauna da área do Curimataú, Paraíba. In: ARAÚJO, F. S.; RODAL, M. J. N.; BARBOSA, M. R. V. (Ed.). Análise das variações da biodiversidade do Bioma Caatinga: suporte a estratégias regionais de conservação. Brasília: Ministério do Meio Ambiente, 2005. p. 264-280.

BÉRNILS, R. S.; COSTA, H. C. (Org.). Brazilian reptiles - List of species. 2012. Disponível em: $<$ http://www.sbherpetologia.org. br/?page_id=629>. Acesso em: 11 ago. 2012.

CARVALHO, C. M.; VILAR, J. C.; OLIVEIRA. F. F. Répteis e Anfíbios. Parque Nacional Serra da Itabaiana - Levantamento da Biota. In: CARVALHO, C. M.; VILAR, J. C. (Coord.). Biologia experimental geral e experimental - UFS. Aracaju: IBAMA, 2005. p. 39-61.

CENCHIN, S. Z.; MARTINS, M. Eficiência das armadilhas de queda (pitfall traps) em amostragens de anfíbios e répteis no Brasil. Revista Brasileira de Zoologia, Curitiba, v. 17, n. 3, p. 729-740, 2000.

DIAS, E. J. R.; ROCHA, C. F. D. Ecpleopus gaudichaudi Duméril and Bibron, 1839 (Squamata: Gymnophthalmidae) and Psychosaura agmosticha (Rodrigues, 2000) (Squamata: Scincidae): Distribution extension and new records from Atlantic Forest in Bahia state, Brazil. Check List, Rio Claro, v. 9, n. 3, p. 607-609, 2013.

FREIRE, E. X. F.; SKUK, G; KOLODIUK, M. F.; RIBEIRO, L. B.; MAGGI, B. S.; RODRIGUES, L. S.; VIEIRA, W. L. S.; FALCÃO, A. C. G. P. Répteis squamata das caatingas do seridó do Rio Grande do Norte e do cariri da Paraíba: síntese do conhecimento atual e perspectivas. In: FREIRE, E. X. F. (Ed.). Recursos naturais das caatingas: uma visão multidisciplinar. Natal: EDUFRN, 2009. p. 51-84.

FREITAS, M. A.; SILVA, T. F. S. A. Guia ilustrado-A herpetofauna das caatingas e áreas de altitudes do nordeste brasileiro (Coleção Manuais de Campo USEB). Vol. 2. Pelotas: USEB, 2006. 384 p.

HEDGES, B. S.; CONN, E. C. A new skink fauna from Caribbean islands (Squamata, Mabuyidae, Mabuyinae). Zootaxa, Auckland, v. 3288, p. 1-244, 2012.

JORGE, J. S.; FREIRE, E. M. X. Mabuya agmosticha (NCN) Geographic distribution. Herpetological Review, St. Louis, v. 41, p. 512-513, 2010.

MOURA, G. J. B.; FREIRE, E. M. X.; SANTOS, E. M.; MORAIS, Z. M. B.; LINS, E. A. M.; ANDRADE, E. V. E.; FERREIRA, J. D. C. Distribuição geográfica dos répteis do estado de Pernambuco. In: MOURA, G. J. B.; SANTOS, E. M.; OLIVEIRA, M. A. B.; CABRAL, M. C. C. (Ed.). Herpetologia no Estado de Pernambuco. Brasília: Ministério do Meio Ambiente, 2011. p. 5084.

RODRIGUES, M. T. A new species of Mabuya (Squamata: Scindidae) from the semi-arid Caatingas of northeastern Brazil. Papéis Avulsos de Zoologia, São Paulo, v. 41, n. 21, p. 313-328, 2000.

RODRIGUES, M. T. Herpetofauna da Caatinga. In: LEAL, I. R.; TABARELlI, I. R. M.; SILVA, J. M. C. (Ed.). Ecologia e conservação da Caatinga. Recife: Editora Universitária, Universidade Federal de Pernambuco, 2003. p. 181-236.

VANZOLINI, P. E. Paleoclimas e especiação em animais na América do Sul. São Paulo: Associação Brasileira de Estudos do Quaternário, 1986.25 p. 